\title{
An SPSS Macro to Compute Confidence Intervals for Pearson's Correlation
}

\author{
Bruce Weaver ${ }^{\mathrm{I}}$, a, Ray Koopman ${ }^{\mathrm{b}}$ \\ ${ }^{a}$ Human Sciences Division, Northern Ontario School of Medicine, and Centre for Research on Safe Driving, Lakehead University \\ b Department of Psychology, Simon Fraser University
}

\begin{abstract}
In many disciplines, including psychology, medical research, epidemiology and public health, authors are required, or at least encouraged to report confidence intervals (CIs) along with effect size estimates. Many students and researchers in these areas use IBM-SPSS for statistical analysis. Unfortunately, the CORRELATIONS procedure in SPSS does not provide CIs in the output. Various work-around solutions have been suggested for obtaining CIs for rho with SPSS, but most of them have been suboptimal. Since release 18, it has been possible to compute bootstrap CIs, but only if users have the optional bootstrap module. The !rhoCI macro described in this article is accessible to all SPSS users with release 14 or later. It directs output from the CORRELATIONS procedure to another dataset, restructures that dataset to have one row per correlation, computes a CI for each correlation, and displays the results in a single table. Because the macro uses the CORRELATIONS procedure, it allows users to specify a list of two or more variables to include in the correlation matrix, to choose a confidence level, and to select either listwise or pairwise deletion. Thus, it offers substantial improvements over previous solutions to the problem of how to compute CIs for rho with SPSS..
\end{abstract}

Keywords $\approx$ Correlation, confidence interval, SPSS

Đbweaver@lakeheadu.ca

\section{Introduction}

One of the recommendations from the American Psychological Association's Task Force on Statistical Inference (TFSI) was that "interval estimates [i.e., confidence intervals] should be given for any effect sizes involving principal outcomes" (Wilkinson \& APA Task Force on Statistical Inference, 1999). The report goes on to mention correlations specifically: "Provide intervals for correlations and other coefficients of association or variation whenever possible."

Many medical and health-related journals also require authors to report confidence intervals (CIs) for effect sizes, including correlations. For example, the website of the International Committee of Medical Journal Editors (ICMJE) includes Uniform Requirements for Manuscripts (URM) submitted to biomedical journals. The URM includes this statement: "When possible, quantify findings and present them with appropriate indicators of measurement error or uncertainty (such as confidence intervals)" (International Committee of Medical Journal Editors, n.d.).

IBM-SPSS is a very popular statistical package that is used by many students and researchers in psychology, epidemiology, public health and related disciplines. Unfortunately, the CORRELATIONS procedure in SPSS does not include CIs as part of the output. Therefore, how to obtain the CI for rho is a rather frequently asked question in SPSS discussion forums. ${ }^{1}$ One solution is to use the syntax found in this Technote on the IBM-SPSS website: http://www-01.ibm.com/support/docview. wss?uid=swg21478368. The Technote syntax works insofar as it provides correct results, but is less than ideal for many SPSS users. The main issue is that it does not work on raw data. Rather, users must compute the correlations for which they want CIs, and then supply the values of $r$ and $n$, either by entering them into a dataset, or by using a DATA LIST command in a syntax window.

Two other methods that have been proposed were discussed in this thread from the SPSSX-L mailing list: http://spssx-discussion.1045642.n5.nabble.com/SPSSsyntax-for-CI-for-correlation-td1069009.html. One suggestion was to use Andrew Hayes' "rci" macro. It uses the SPSS MATRIX language to compute the 95\% CI for rho. Unlike the SPSS Technote syntax, it has the advantage of working on raw data. But it does have

\footnotetext{
1 The Greek letter rho ( $\rho)$ is used to symbolize the parameter corresponding to Pearson's $r$. CIs are for parameters, not for the statistics that estimate those parameters. Therefore, the CI for Pearson's correlation is properly described as the CI for rho, not the CI for $r$.
} 
some limitations. First, it takes only two variables at a time. Therefore, if one wants CIs for a correlation matrix with three or more variables, the macro must be called once for each pair of variables. And to obtain listwise deletion, one must first set a filter to ensure that only cases with valid data for all of the variables are included. Second, Hayes' macro is hard coded to compute the $95 \%$ CI. Users comfortable with programming could modify the syntax easily enough to use other confidence levels, but it would be preferable to have a macro argument that specifies the desired confidence level. And finally, the URL for Hayes' macro shown in the SPSSX-L discussion is no longer active, so finding it may be difficult.

The IBM-SPSS Technote syntax and Hayes' rci macro both compute the $\mathrm{CI}$ for rho using the conventional method (see Beaulieu-Prévost, 2006 and Fisher, 1925). First, Fisher's $r$-to- $Z$ transformation is applied to the sample correlation (see Equation 1).

$$
Z_{r}=\operatorname{arctanh}(r)=(0.5) \log _{e}\left(\frac{1+r}{1-r}\right)
$$

Then the limits of the CI are computed on the transformed scale (see Equation 2, where $z_{\alpha / 2}$ is the critical value of $z$ for a two-tailed $\alpha$-level test).

$$
100(1-\alpha) \% \text { CI for } Z_{\rho}=Z_{r} \pm \frac{z_{\alpha / 2}}{\sqrt{n-3}}
$$

And finally, the lower and upper limits of the CI are transformed back to the original scale (Equation 3$){ }^{2}$

$$
r=\tanh \left(Z_{r}\right)=\frac{e^{Z_{r}}-e^{-Z_{r}}}{e^{Z_{r}}+e^{-Z_{r}}}
$$

Another option discussed in the SPSSX-L thread was to perform simple linear regression using $z$-scores rather than the original raw scores-see this video, for example: $\quad$ http://www.youtube.com/watch?v=dSoWqDyT4E. This approach is based on the fact that

\footnotetext{
${ }^{2}$ The $\tanh$ in Equation 3 is the hyperbolic tangent, and the arctanh in Equation 1 is the inverse hyperbolic tangent (sometimes symbolized as atanh or $\left.\tanh h^{-1}\right)$. These functions are not directly available in SPSS, so it is customary to use expressions to the right in Equations 1 and 3 when computing the CI for rho. However, the idf.logistic and cdf.logistic functions, which are available in SPSS, can be used to compute $\operatorname{arctanh}$ and tanh as follows: $\operatorname{arctanh}(r)=$ $.5 \times$ idf.logistic $((1+r) / 2,0,1) ; \tanh \left(\mathrm{Z}_{r}\right)=2 \times$ cdf.logistic $\left(2 \mathrm{Z}_{r}, 0,1\right)-1$. More details about the idf.logistic and cdf.logistic functions can be found in the IBM-SPSS Command Syntax Reference manual.
}

conceptually, Pearson's $r$ is equal to the slope from a simple linear regression model using standardized $X$ and $Y$ values. And of course, the REGRESSION procedure does provide a confidence interval for the slope. The $\mathrm{CI}$ from regression with standard scores is $r \pm t_{\alpha / 2} \sqrt{\left(1-r^{2}\right) /(n-2)}$. But those limits are necessarily wrong for two reasons: First, they are symmetric around $r$, second, they can exceed 1 and -1 . The correct interval, on the other hand is always wider on the side of $r$ toward zero than on the side away from zero; and it always has limits that fall within the range -1 to 1 . Therefore, the $\mathrm{CI}$ for the slope from a simple linear regression with standard scores must not be used as the $\mathrm{CI}$ for rho.

The aforementioned video also shows how to compute bootstrap CIs for rho using SPSS Amos, a program for structural equations modeling (SEM). However, the main SPSS Statistics program can also compute bootstrap CIs for rho, provided one has access to the optional bootstrap module (first available in release 18). As suggested in the video, bootstrap CIs are legitimate, and may be preferable to CIs computed using standard asymptotic methods when distributional assumptions for the latter are too grossly violated. However, the bootstrap module is optional, so not all SPSS users have access to it. The macro described below, on the other hand, requires only the statistics base module and the ability to work with multiple datasets. Therefore, it is accessible to all SPSS users who have release 14 or later.

\section{Description of the !rhoCI macro ${ }^{3}$}

The limitations of currently available methods for computing CIs for rho motivated us to write a new, more flexible and user-friendly SPSS macro. The !rhoCI macro uses the /MATRIX OUT subcommand of the CORRELATIONS procedure to write the correlations and sample sizes to another dataset. Using matrix output from CORRELATIONS has several advantages: 1) The macro works on raw data; 2 ) one can have as many variables as desired; and 3) one can choose either LISTWISE or PAIRWISE deletion. (If LISTWISE deletion is selected, each correlation is computed using only

\footnotetext{
${ }^{3}$ Macro names do not have to begin with an exclamation mark, so we could have called our macro rhoCI. We called it !rhoCI in accordance with a practice recommended by Raynald Levesque, the owner of the well-known SPSS Tools website (www.spsstools.net). Levesque's reason for starting macro names with an exclamation mark is that this makes it very easy for someone viewing a syntax file to recognize macros. Furthermore, all macros that follow this naming convention can be found quickly by searching for the explanation point.
} 
Table 1 - Description of the five !rhoCI macro arguments.

\begin{tabular}{|c|c|c|}
\hline Argument $^{a}$ & Mandatory? & Function \\
\hline DataSetName & Yes & $\begin{array}{l}\text { To specify the name of the dataset containing the raw data } \\
\text { used for computing the correlations (e.g., DataSetName = } \\
\text { RawData) }\end{array}$ \\
\hline Vars & Yes & $\begin{array}{l}\text { To provide a list of variables to be correlated; can use the } \\
\text { T0 convention (e.g., /Vars = v1 T0 v10) }\end{array}$ \\
\hline ConfidenceLevel & No & $\begin{array}{l}\text { To specify the desired confidence level (e.g., } \\
\text { /ConfidenceLevel = 99); if omitted, the default value is } 95\end{array}$ \\
\hline ListWise & No & $\begin{array}{l}\text { An indicator variable for LISTWISE deletion; e.g., } \\
/ \text { ListWise }=1 \text { to select listwise deletion, or } / \text { Listwise }=0 \text { to } \\
\text { select pairwise deletion; if ListWise is omitted, the default } \\
\text { value is } 0 \text { (pairwise) }\end{array}$ \\
\hline CorrMat & No & $\begin{array}{l}\text { An indicator variable for display of the correlation matrix } \\
\text { produced by the CORRELATIONS procedure; e.g., } \\
\text { /CorrMat=1 to display the correlation matrix, /CorrMat }=0 \\
\text { to suppress its display; if CorrMat is omitted, the default } \\
\text { value is } 0 \text { (no correlation matrix) }\end{array}$ \\
\hline
\end{tabular}

a Note that like other SPSS syntax, the macro arguments are not case-sensitive.

cases that have valid data for all variables in the list, so the sample size is the same for all correlations. If PAIRWISE deletion is selected, all cases with valid data for each pair of variables are used, so the sample size is not necessarily the same for each correlation.)

The macro has two mandatory and three optional arguments (see Table 1). The first mandatory argument, DataSetName, is the name of the dataset containing the raw data to be used for the computing the correlations (e.g., DataSetName $=$ raw). ${ }^{4}$ (Dataset names are shown in square brackets beside the file name in the top left corner of the Data Editor window.) The second mandatory argument, Vars, is a list of variables to include in the correlation matrix. It can be set to a list of individual variable names (e.g., Vars = educ paeduc maeduc speduc), or one can use the TO convention if the variables are contiguous in the data file (e.g.,Vars $=$ educ T0 speduc). ${ }^{5}$ The third argument, ConfidenceLevel, allows the user to specify the desired confidence level (e.g., ConfidenceLevel $=95$, or ConfidenceLevel $=99$, etc.). The fourth argument, ListWise, is an indicator variable for LISTWISE deletion (ListWise $=1$ to choose LISTWISE, ListWise $=0$ to choose PAIRWISE). And the final argument, CorrMat, is

\footnotetext{
${ }^{4}$ It is necessary to have a dataset name associated with the file containing the raw data if one wishes that dataset to remain open for subsequent use after calling the macro.

5 The key word WITH cannot be used, however, because CORRELATIONS cannot write matrix output in that case.
}

an indicator for showing the matrix produced by the CORRELATIONS command in the output (CorrMat $=1$ to show the correlation matrix, CorrMat $=0$ to suppress it). ${ }^{6}$ As noted above, the first two macro arguments (DataSetName and Vars) are mandatory. The other arguments can be omitted, but will then default to the following values: ConfidenceLevel $=95$, ListWise $=0$ and CorrMat $=0$.

$$
\begin{aligned}
100(1-\alpha) \% & \text { CI for } \rho=\tanh \left(Z_{r} \pm d\right) \\
& =\frac{\tanh \left(Z_{r}\right) \pm \tanh (d)}{1 \pm \tanh \left(Z_{r}\right) \times \tanh (d)} \\
& =\frac{r \pm \tanh (d)}{1 \pm r \times \tanh (d)}
\end{aligned}
$$

The macro computes the $\mathrm{CI}$ for rho as shown in Equation 4, where $d=z_{\alpha / 2} / \sqrt{n-3}$. This simple variation on the usual method uses an algebraic shortcut based on the standard hyperbolic identity, $\tanh (a+b) \equiv[\tanh (a)+\tanh (b)] /[1+\tanh (a) \times$ $\tanh (b)]$ (see Equation 4.5.26 in Abramowitz \& Stegun, 1972). Note that the rightmost expression in Equation 4 works even when $r=1$ or -1 . Equation 1 , on the other hand, breaks down in both of those cases: When $r=1$, $(1+r) /(1-r)$ results in division by zero; and when $r=$

\footnotetext{
${ }^{6}$ We included the option of displaying the correlation matrix from CORRELATIONS so that readers who wish to do so can verify that the correlations and $p$-values in the macro's summary table are correct.
} 


\begin{tabular}{|c|c|c|c|c|c|c|c|c|}
\hline \multicolumn{9}{|c|}{ Pearson correlations with $95 \%$} \\
\hline & $X$ & $\mathrm{Y}$ & $r$ & Lower & Upper & $p$ & $\mathrm{n}$ & Notes \\
\hline 1 & age & age & 1.000 & & & & 497 & \\
\hline 2 & age & educ & -.049 & -.136 & .040 & .281 & 496 & \\
\hline 3 & age & speduc & .034 & -.105 & .172 & .634 & 200 & \\
\hline 4 & age & childs & .319 & .238 & .396 & .000 & 496 & \\
\hline 5 & educ & age & -.049 & -.136 & .040 & .281 & 496 & \\
\hline 6 & educ & educ & 1.000 & & & & 499 & \\
\hline 7 & educ & speduc & .598 & .501 & .680 & .000 & 200 & \\
\hline 8 & educ & childs & -.256 & -.336 & -.172 & .000 & 498 & \\
\hline 9 & speduc & age & .034 & -.105 & .172 & .634 & 200 & \\
\hline 10 & speduc & educ & .598 & .501 & .680 & .000 & 200 & \\
\hline 11 & speduc & speduc & 1.000 & & & & 201 & \\
\hline 12 & speduc & childs & -.120 & -.254 & .018 & .089 & 201 & \\
\hline 13 & childs & age & .319 & .238 & .396 & .000 & 496 & \\
\hline 14 & childs & educ & -.256 & -.336 & -.172 & .000 & 498 & \\
\hline 15 & childs & speduc & -.120 & -.254 & .018 & .089 & 201 & \\
\hline 16 & childs & childs & 1.000 & & & & 499 & \\
\hline
\end{tabular}

Figure 1 - Output for Example 1. The three optional macro arguments were omitted, so they took their default values: Confidencelevel $=95$, ListWise $=0$ and CorrMat $=0$. ListWise $=0$ indicates use of pairwise deletion, and CorrMat $=0$ suppresses display of the correlation matrix generated by the CORRELATIONS procedure.

$-1, \log _{\mathrm{e}}((1+r) /(1-r))=\log _{\mathrm{e}}(0)$, which is undefined.

\section{Examples}

Before the !rhoCI macro can be used, the macro definition (shown in the appendix) must be run. A convenient way to do this is via the INSERT FILE command, as follows:

INSERT FILE =

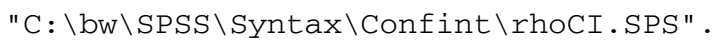

Users will have to modify the path to match where the rhoCI.SPS syntax file is stored on their computers. Note too that macro definitions remain active until you exit SPSS, so you do not have to run the macro definition before each call to the macro. Running it once per SPSS session will suffice.

The examples shown below were generated using syntax file rhoCI_examples.SPS, which can be downloaded from the first author's website (see the link provided in the appendix) and as part of the supplementary material on the journal's website. ${ }^{7} \mathrm{We}$ used only the first 500 records from the survey_sample.sav data file that can be found in the Samples sub-folder where IBM-SPSS is installed. Often, one uses all of the data in a file when computing

7 The rhoCI_examples.SPS file includes other examples not shown here. For example, it shows how to compute bootstrap CIs for rho. correlations. We used only the first 500 records in this case because when we used all records, the sample size was so large that all $p$-values were less than .001, and all of the CIs were extremely narrow. By using only the first 500 records, we obtained a situation that is more typical of what most readers will see when analyzing their own data-i.e., some correlations are statistically significant, whereas others are not, and variation of width in the CIs is more easily seen.

The first example shown below uses only the first two mandatory macro arguments, DataSetName and Vars. Because the optional arguments are omitted, they take their default values: ConfidenceLevel $=95$, ListWise $=0$ and CorrMat $=0$. The output for Example 1 is shown in Figure 1.

* Example 1.

! rhocI DatasetName = raw

/Vars = age educ speduc childs.

The second example computes uses the same variable list as Example 1, but computes $99 \%$ rather than 95\% CIs, and it uses listwise deletion rather than the default pairwise deletion. The results for this macro call are shown in Figure 2.

\footnotetext{
* Example 2.

! rhocI DatasetName = raw

/Vars = age educ speduc childs
} 


\begin{tabular}{lllllllll}
\multicolumn{10}{c}{ Pearson correlations with 99\% confidence intervals* } \\
\hline \hline 1 & $\mathrm{X}$ & $\mathrm{Y}$ & $\mathrm{r}$ & Lower & Upper & $\mathrm{p}$ & $\mathrm{n}$ & Notes \\
2 & age & age & 1.000 & & & & 199 \\
3 & age & educ & .057 & -.126 & .237 & .421 & 199 \\
4 & age & childs & .324 & .151 & .478 & .000 & 199 \\
5 & educ & age & .057 & -.126 & .237 & .421 & 199 \\
6 & educ & educ & 1.000 & & & & 199 \\
7 & educ & speduc & .597 & .465 & .702 & .000 & 199 \\
8 & educ & childs & -.172 & -.344 & .010 & .015 & 199 \\
9 & speduc & age & .038 & -.144 & .219 & .590 & 199 \\
10 & speduc & educ & .597 & .465 & .702 & .000 & 199 \\
11 & speduc & speduc & 1.000 & & & & 199 \\
12 & speduc & childs & -.120 & -.295 & .063 & .092 & 199 \\
13 & childs & age & .324 & .151 & .478 & .000 & 199 \\
14 & childs & educ & -.172 & -.344 & .010 & .015 & 199 \\
15 & childs & speduc & -.120 & -.295 & .063 & .092 & 199 \\
16 & childs & childs & 1.000 & & & & 199 \\
\hline \multirow{2}{*}{ With }
\end{tabular}

Figure 2. Output for Example 2. ConfidenceLevel was set to 99 and ListWise to 1. Thus $99 \%$ CIs are shown, and the sample size is the same for all correlations.

/ Confidencelevel $=99$

/ListWise $=1$.

Example 3 demonstrates the use of the TO convention on the Vars argument. And by setting the CorrMat argument to 1 , it tells the macro to display the usual correlation matrix generated by the CORRELATIONS procedure. Confidencelevel and ListWise ore omitted, so they take their default values (i.e., 95 and 0). The results are show in Figure 3.

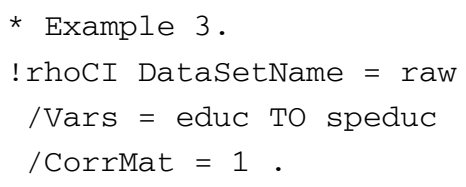

Example 4 demonstrates the use of the macro when the sample size is quite small. This was done to illustrate the various warning messages that may appear in the Notes field of the summary table when the sample size is small. The results are show in Figure 4.

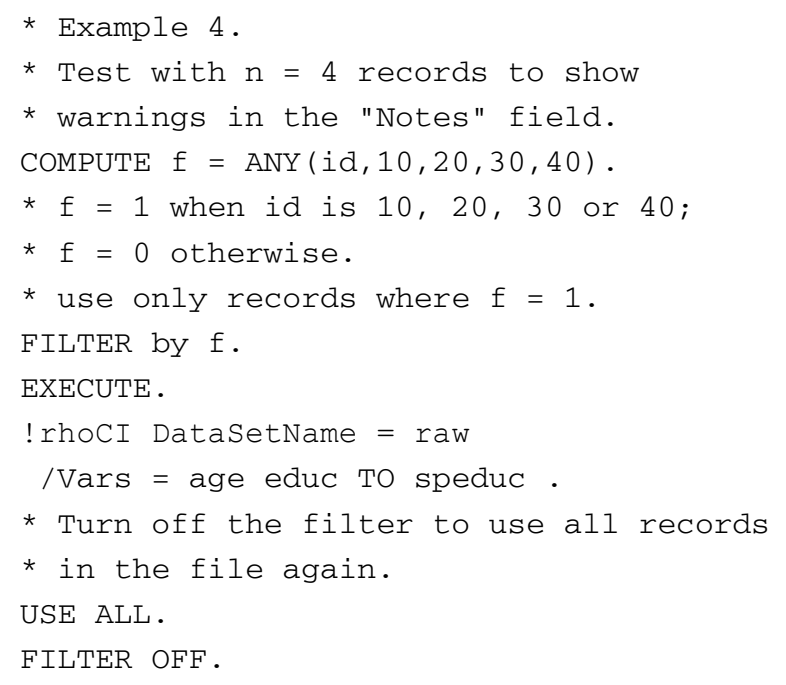

As shown in Figure 4, the three possible warning messages that can appear in the Notes field are:

- $n<3: p$ and CI not computed

$-n<4:$ CI not computed

$-n<10$ : Normal approximation is poor 
Pearson correlations with $95 \%$ confidence intervals*

\begin{tabular}{|c|c|c|c|c|c|c|c|c|}
\hline & $x$ & $Y$ & $r$ & Lower & Upper & $p$ & $\mathrm{n}$ & Notes \\
\hline 1 & educ & educ & 1.000 & & & & 499 & \\
\hline 2 & educ & paeduc & .379 & .286 & .464 & .000 & 358 & \\
\hline 3 & educ & maeduc & .290 & .201 & .374 & .000 & 433 & \\
\hline 4 & educ & speduc & .598 & .501 & .680 & .000 & 200 & \\
\hline 5 & paeduc & educ & .379 & .286 & .464 & .000 & 358 & \\
\hline 6 & paeduc & paeduc & 1.000 & & & & 359 & \\
\hline 7 & paeduc & maeduc & .644 & .577 & .703 & .000 & 338 & \\
\hline 8 & paeduc & speduc & .411 & .273 & .533 & .000 & 159 & \\
\hline 9 & maeduc & educ & .290 & .201 & .374 & .000 & 433 & \\
\hline 10 & maeduc & paeduc & .644 & .577 & .703 & .000 & 338 & \\
\hline 11 & maeduc & maeduc & 1.000 & & & & 434 & \\
\hline 12 & maeduc & speduc & .323 & .185 & .449 & .000 & 179 & \\
\hline 13 & speduc & educ & .598 & .501 & .680 & .000 & 200 & \\
\hline 14 & speduc & paeduc & .411 & .273 & .533 & .000 & 159 & \\
\hline 15 & speduc & maeduc & .323 & .185 & .449 & .000 & 179 & \\
\hline 16 & speduc & speduc & 1.000 & & & & 201 & \\
\hline
\end{tabular}

* With PAIRWISE deletion.

\begin{tabular}{|c|c|c|c|c|c|}
\hline \multicolumn{6}{|c|}{ Correlations } \\
\hline & & $\begin{array}{c}\text { Highest } \\
\text { year of } \\
\text { school } \\
\text { completed }\end{array}$ & $\begin{array}{c}\text { Highest } \\
\text { year school } \\
\text { completed, } \\
\text { father }\end{array}$ & $\begin{array}{c}\text { Highest } \\
\text { year school } \\
\text { completed, } \\
\text { mother }\end{array}$ & $\begin{array}{c}\text { Highest } \\
\text { year school } \\
\text { completed, } \\
\text { spouse }\end{array}$ \\
\hline \multirow{3}{*}{$\begin{array}{l}\text { Highest year of } \\
\text { school completed }\end{array}$} & Pearson Correlation & 1 & $.379^{*}$ & $.290^{*}$ & $.598^{* *}$ \\
\hline & Sig. (2-tailed) & & .000 & .000 & .000 \\
\hline & $\mathrm{N}$ & 499 & 358 & 433 & 200 \\
\hline \multirow{3}{*}{$\begin{array}{l}\text { Highest year school } \\
\text { completed, father }\end{array}$} & Pearson Correlation & $.379^{* *}$ & 1 & $644^{*}$ & $.411^{*}$ \\
\hline & Sig. (2-tailed) & .000 & & .000 & .000 \\
\hline & $\mathrm{N}$ & 358 & 359 & 338 & 159 \\
\hline \multirow{3}{*}{$\begin{array}{l}\text { Highest year school } \\
\text { completed, mother }\end{array}$} & Pearson Correlation & $.290^{\circ}$ & $.644^{*}$ & 1 & $.323^{*}$ \\
\hline & Sig. (2-tailed) & .000 & .000 & & .000 \\
\hline & $\mathrm{N}$ & 433 & 338 & 434 & 179 \\
\hline \multirow{3}{*}{$\begin{array}{l}\text { Highest year school } \\
\text { completed, spouse }\end{array}$} & Pearson Correlation & $.598^{*}$ & $.411^{*}$ & $.323^{*}$ & 1 \\
\hline & Sig. (2-tailed) & .000 & .000 & .000 & \\
\hline & $\mathrm{N}$ & 200 & 159 & 179 & 201 \\
\hline
\end{tabular}

${ }^{* *}$. Correlation is significant at the 0.01 level (2-tailed).

Figure 3 - Output for Example 3. CorrMat was set to 1, so the correlation matrix generated by the CORRELATIONS procedure is displayed. ConfidenceLevel and ListWise were omitted, so they took their default values of 95 and 0 respectively.

Computation of the $p$-value entails using a $t$ distribution with degrees of freedom equal to $n-2$; and the standard error of $Z_{r}$ (which is used in computing the CI for $r h o$ ) has $\sqrt{n-3}$ in the denominator. Thus computation of the $p$-value requires $n \geq 3$ and computation of the $\mathrm{CI}$ for rho requires $n \geq 4$. The warning about the normal approximation being poor for $n<10$ is based on Kirk's (2007) discussion of CIs for rho.

\section{Summary}

In many disciplines, including psychology, medical research, public health and epidemiology, authors are asked to report CIs for effect size estimates. IBM-SPSS does not provide CIs for Pearson's correlation in the output from its CORRELATIONS procedure. Various 
Pearson correlations with $95 \%$ confidence intervals*

\begin{tabular}{|c|c|c|c|c|c|c|c|}
\hline & $\mathrm{X}$ & $\overline{\mathrm{Y}}$ & $\bar{r}$ & Lower & Upper & $\bar{p}$ & Notes \\
\hline 1 & age & age & 1.000 & & & & 4 \\
\hline 2 & age & educ & -.159 & -.972 & .947 & .841 & $4 n<10$ : Normal approximation is poor \\
\hline 3 & age & paeduc & .012 & -.960 & .962 & .988 & $4 n<10$ : Normal approximation is poor \\
\hline 4 & age & maeduc & -1.000 & & & .011 & $3 \mathrm{n}<4$ : Cl not computed \\
\hline 5 & age & speduc & 1.000 & & & & $2 \mathrm{n}<3$ : $\mathrm{p}$ and $\mathrm{Cl}$ not computed \\
\hline 6 & educ & age & -.159 & -.972 & .947 & .841 & $4 \mathrm{n}<10$ : Normal approximation is poor \\
\hline 7 & educ & educ & 1.000 & & & & 4 \\
\hline 8 & educ & paeduc & .971 & .153 & .999 & .029 & $4 n<10$ : Normal approximation is poor \\
\hline 9 & educ & maeduc & .982 & & & .121 & $3 \mathrm{n}<4$ : Cl not computed \\
\hline 10 & educ & speduc & 1.000 & & & & $2 \mathrm{n}<3$ : $\mathrm{p}$ and $\mathrm{Cl}$ not computed \\
\hline 11 & paeduc & age & .012 & -.960 & .962 & .988 & $4 n<10$ : Normal approximation is poor \\
\hline 12 & paeduc & educ & .971 & .153 & .999 & .029 & $4 n<10$ : Normal approximation is poor \\
\hline 13 & paeduc & paeduc & 1.000 & & & & 4 \\
\hline 14 & paeduc & maeduc & .866 & & & .333 & $3 \mathrm{n}<4$ : Cl not computed \\
\hline 15 & paeduc & speduc & 1.000 & & & & $2 \mathrm{n}<3: \mathrm{p}$ and $\mathrm{Cl}$ not computed \\
\hline 16 & maeduc & age & -1.000 & & & .011 & $3 \mathrm{n}<4$ : Cl not computed \\
\hline 17 & maeduc & educ & .982 & & & .121 & $3 \mathrm{n}<4$ : Cl not computed \\
\hline 18 & maeduc & paeduc & .866 & & & .333 & $3 \mathrm{n}<4$ : Cl not computed \\
\hline 19 & maeduc & maeduc & 1.000 & & & & 3 \\
\hline 20 & speduc & age & 1.000 & & & & $2 \mathrm{n}<3$ : $\mathrm{p}$ and $\mathrm{Cl}$ not computed \\
\hline 21 & speduc & educ & 1.000 & & & & $2 \mathrm{n}<3$ : $\mathrm{p}$ and $\mathrm{Cl}$ not computed \\
\hline 22 & speduc & paeduc & 1.000 & & & & $2 \mathrm{n}<3$ : $\mathrm{p}$ and $\mathrm{Cl}$ not computed \\
\hline 23 & speduc & speduc & 1.000 & & & & 2 \\
\hline
\end{tabular}

Figure 4 = Output for Example 4. Only four records were used for this example. This was done to illustrate the three types of warning messages that can appear in the Notes field of the macro output table when sample sizes are small.

work-around methods for computing those CIs have been used in the past, but they are all sub-optimal in one way or another. Since release 18, it has been possible to compute bootstrap CIs for rho, but the bootstrap module is optional, and therefore not available to all SPSS users. The !rhoCI macro described in this article is accessible to all SPSS users who have release 14 or later. It allows users to specify a list of variables to include in the correlation matrix, to choose a confidence level, and to select either listwise or pairwise deletion. It also summarizes the output in a single table with one row per correlation. We hope it will prove useful to SPSS users, and that it will help lead to improved reporting of CIs for rho.

\section{Authors' notes and acknowledgments}

The first author thanks Sacha Dubois for reminding him of the usefulness of reading the fine manual (RTFM) when things are not working quite as desired. In particular, Sacha helped me realize that I needed to use variable name index rather than a simple numeric index in the VARSTOCASES command that is a crucial part of the !rhoCI macro. We also thank two anonymous reviewers for their thoughtful and constructive comments on an earlier draft of this article.

\section{References}

Abramowitz, M., \& Stegun, I. A. (1972). Handbook of mathematical functions with formulas, graphs, and mathematical tables (10 th printing, with corrections) National Bureau of Standards Applied Mathematics Series 55.

Beaulieu-Prévost, D. (2006). Confidence intervals: From tests of statistical significance to confidence intervals, range hypotheses and substantial effects. Tutorials in Quantitative Methods for Psychology, 2, 11.

Fisher, R. A. (1925). Statistical methods for research workers. Edinburgh: Oliver and Boyd.

International Committee of Medical Journal Editors. (n.d.) Uniform requirements for manuscripts submitted to biomedical journals: Writing and editing for biomedical publication. Retrieved 31May-2013, from http://www.ICMJE.org. 
Kirk, R. E. (2007). Statistics: An introduction Wadsworth Publishing Company. Wilkinson, L., \& APA Task Force on Statistical Inference.
(1999). Statistical methods in psychology journals: Guidelines and explanations. American Psychologist, 54, 594-604.

\section{Appendix: The !rhoCI Macro Definition}

Before using the !rhoCI macro, users must run the macro definition shown below. (As noted in the article, a convenient way to do that is via the INSERT FILE command.) After running the macro definition, the macro remains available until the SPSS session is terminated. This macro definition, the syntax used to generate the examples reported in the text of the article and a file containing full output for the examples are available as part of the supplementary material on the journal's website. They can also be downloaded from the first author's website. Use the first link below to download the macro definition, the second link to download syntax for the examples, and the third to see the full output from the syntax file.

- https://sites.google.com/a/lakeheadu.ca/bweaver/Home/statistics/files/rhoCI.txt?attredirects=0

- https://sites.google.com/a/lakeheadu.ca/bweaver/Home/statistics/files/rhoCI_examples.txt?attredirects=0

- https://sites.google.com/a/lakeheadu.ca/bweaver/Home/statistics/files/rhoCI_examples.pdf?attredirects=0\&d=1

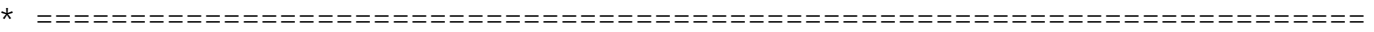

* File: rhocI.SPS.

* Date: 3-Jun-2013.

* Author: Bruce Weaver, bweaver@lakeheadu.ca.

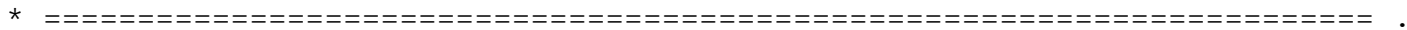

* This file contains an SPSS macro to generate confidence intervals

* for rho, where rho is the parameter corresponding to Pearson's $r$.

* Examples of how to use the macro can be found in syntax file

* "rhocI_examples.sps" .

* The macro uses the following basic equations:

* $\mathrm{Zr}=\operatorname{arctanh}[r]<==>r=\tanh [\mathrm{Zr}]$

* $\mathrm{d}=\mathrm{z}_{-}$alpha / sqrt $[\mathrm{n}-3]$

* $t=\tanh [d]$

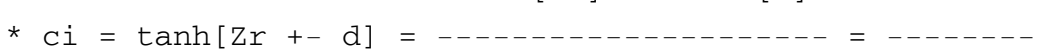

* $1+-\tanh [\mathrm{Zr}] * \tanh [\mathrm{d}] 1+-r^{*}$

* The rightmost term in the last equation works even when $r=+1$ or -1 .

* SPSS has no tanh and arctanh functions.

* HOWEVER, one can use IDF.LOGISTIC and CDF.LOGISTIC as follows:

$* \operatorname{arctanh}[r]=.5 * \ln ((1+r) /(1-r))=.5 * i d f . \operatorname{logistic}((1+r) / 2,0,1)$

$* \tanh [d]=(\exp (2 * d)-1) /(\exp (2 * d)+1)=2 * \operatorname{cof} \cdot \log i \operatorname{stic}(2 * d, 0,1)-1$

* SOURCES :

* http://people.math.sfu.ca/ cbm/aands/page_83.htm (see Equation 4.5.26).

* http://people.math.sfu.ca/ cbm/aands/intro.htm\#001.

* Note that the first two macro arguments, DataSetName and Vars

* are required. The others arguments can be omitted, in which

* case they will take the default values specified below.

* Note too that the macro syntax is NOT case sensitive.

DEFINE !rhOCI

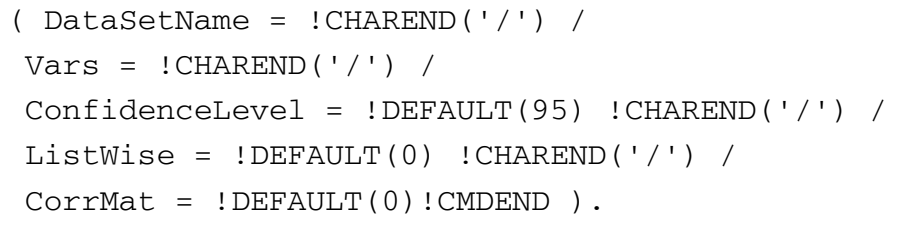




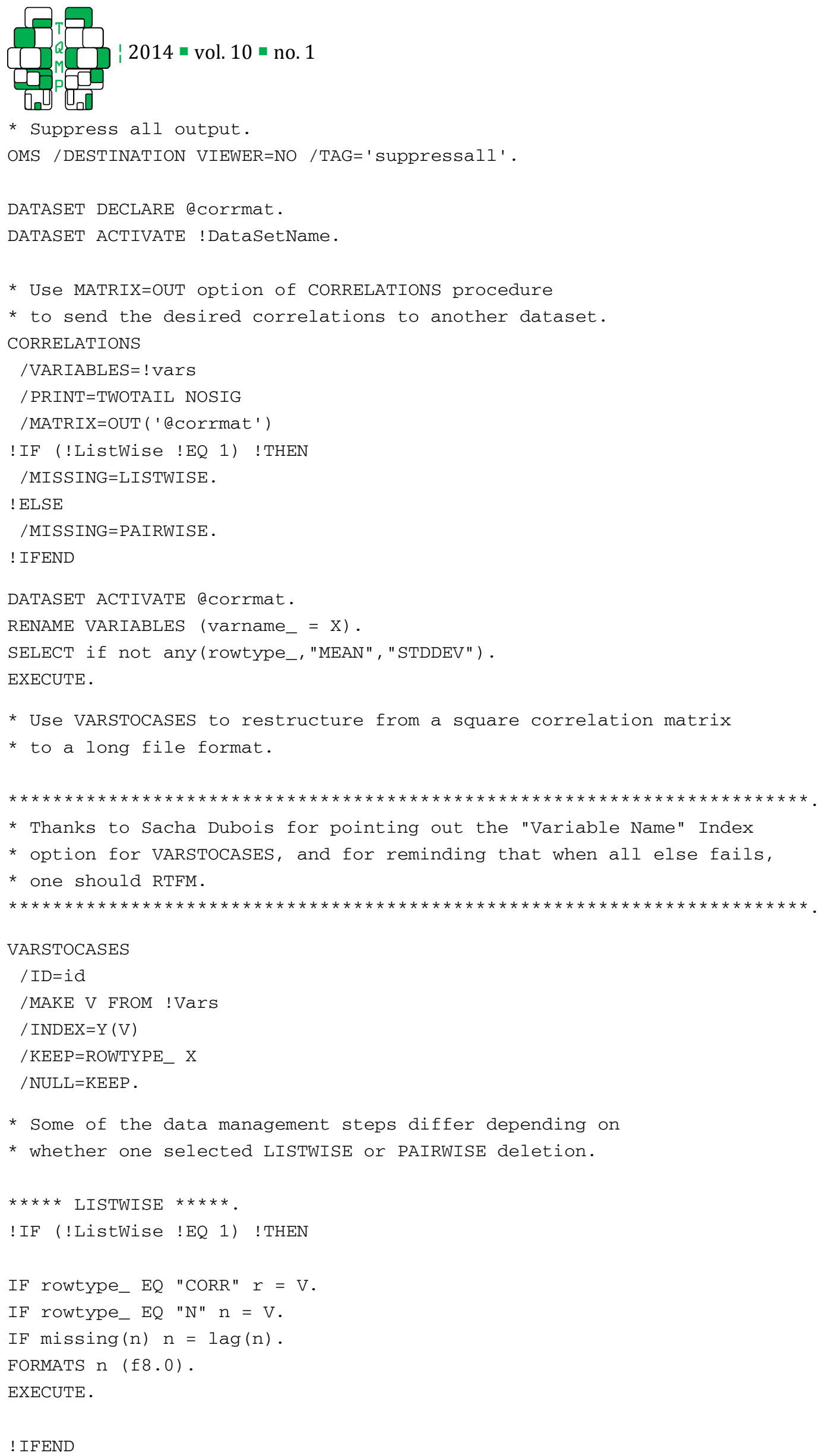




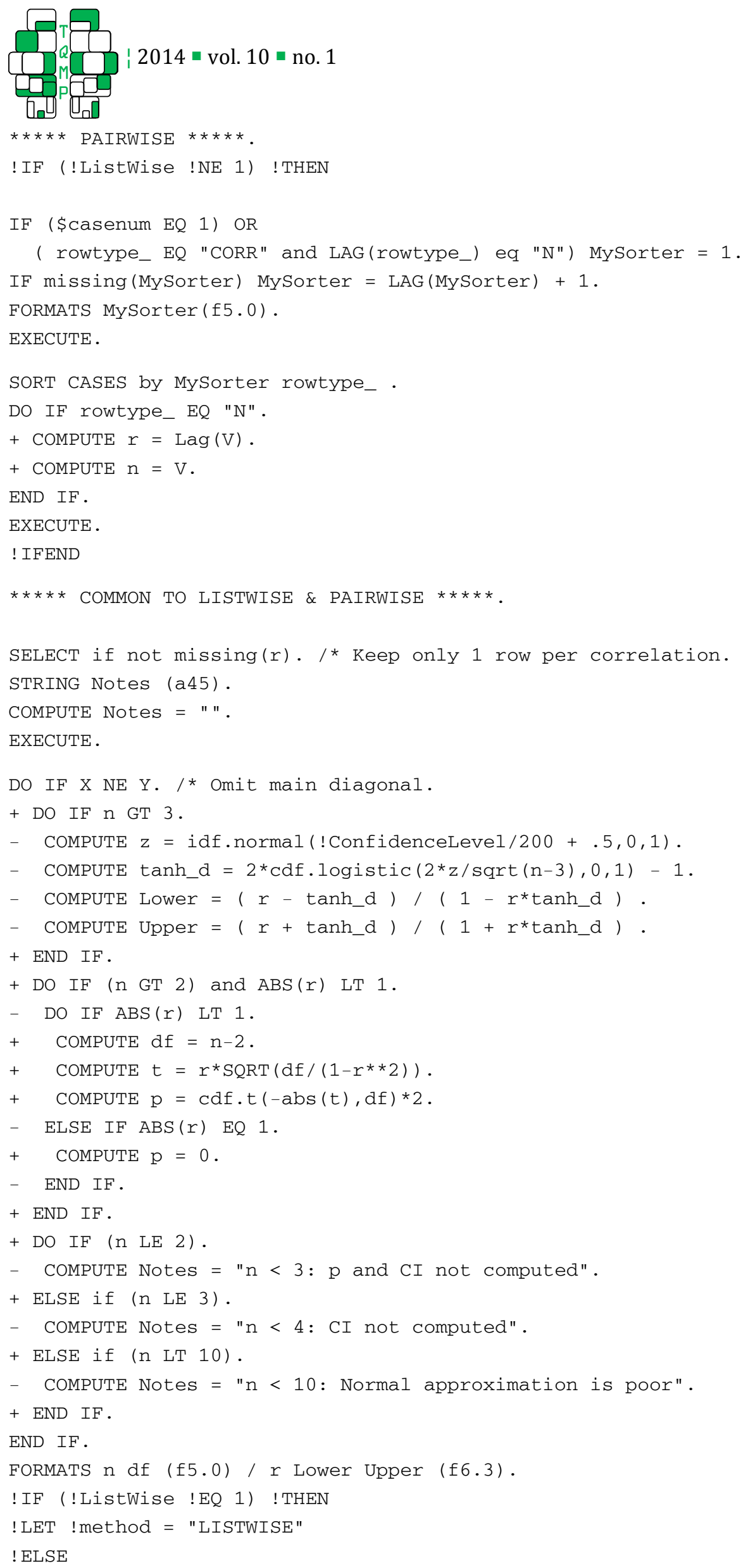




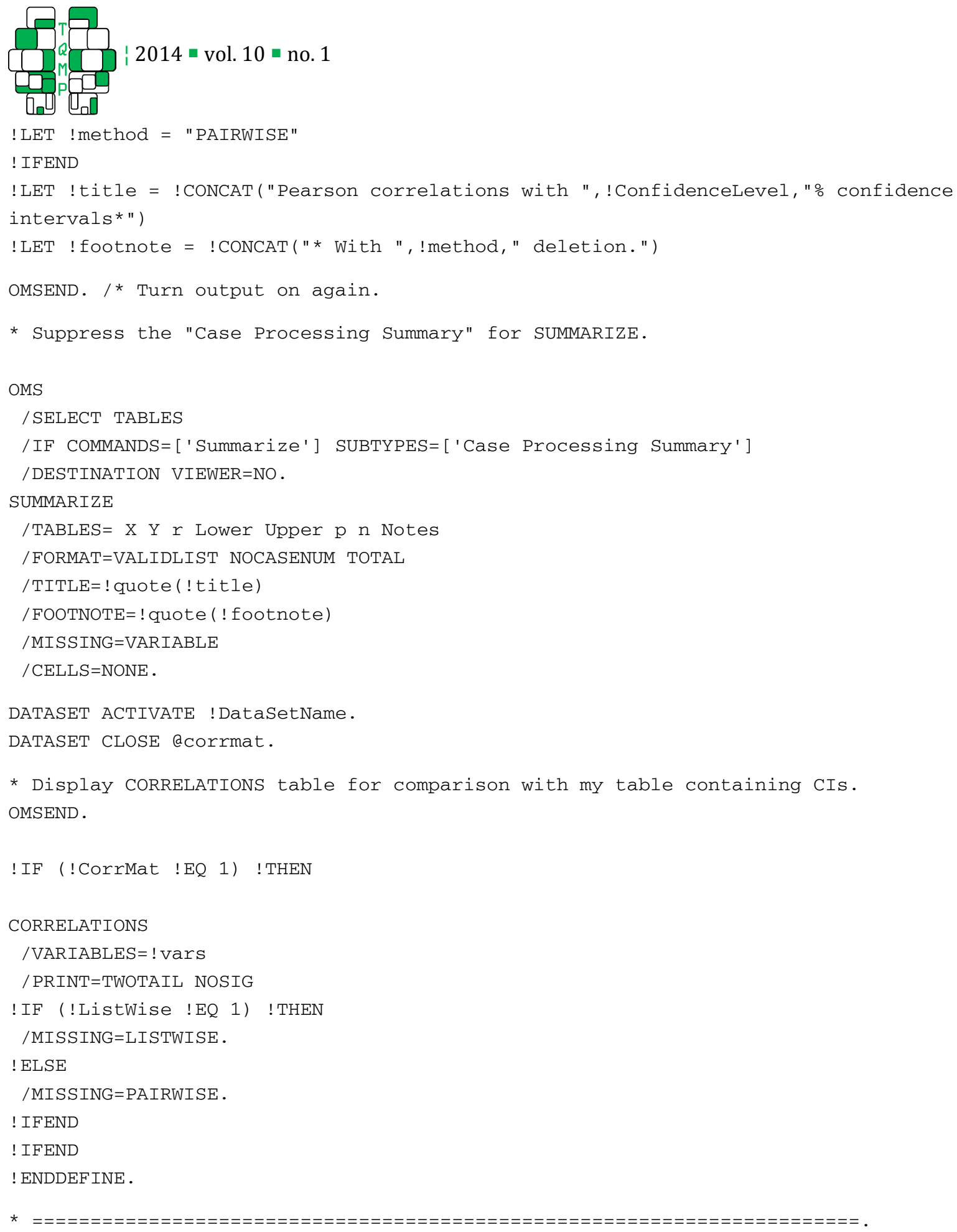

\section{Citation}

Weaver, B., \& Koopman, R. (2014). An SPSS Macro to Compute Confidence Intervals for Pearson's Correlation. The Quantitative Methods for Psychology, 10 (1), 29-39.

Copyright (C) 2014 Weaver and Koopman. This is an open-access article distributed under the terms of the Creative Commons Attribution License (CC BY). The use, distribution or reproduction in other forums is permitted, provided the original author(s) or licensor are credited and that the original publication in this journal is cited, in accordance with accepted academic practice. No use, distribution or reproduction is permitted which does not comply with these terms. 